\title{
Cálculo dos coeficientes de perda de carga, utilizando coeficiente de Reynolds e Bernoulli em um escoamento laminar para o aumento da vazão em um pasteurizador de suco
}

Andresa Morais Aprigio - andressamorais2016@gmail.com Juliana Karine de Moraes Pereira - julianakarinekta@ hotmail.com Tiago Bittencourt Nazaré - tiago@ unis.edu.br

\section{RESUMO}

A produção de alimentos é geralmente praticada em todas as regiões do Brasil, e o maior desafio de todas as empresas é fabricar alimentos saudáveis com o menor risco de contaminação possível. A pasteurização é um dos procedimentos utilizados para diminuição de micro-organismos presentes nos alimentos. Com tudo, o presente estudo teve como objetivo a realização de cálculos mecânicos para o aumento da vazão de um pasteurizador na empresa de suco e polpas Britvic Ebba situada na cidade de Astolfo Dutra - MG, passando sua vazão de 7860L/h para 9000L/h. Foram coletadas informações de tempo, pressão e velocidade decorrentes do processo, todos os dados foram apurados no processo em tempo real de produção. Os resultados encontrados demonstraram que com a pressão do fluido, a velocidade de saída será menor que a velocidade de início, de acordo com a altura que os tubos de envase do pasteurizador se encontram.

Conclui-se que os métodos mecânicos de análises foram efetivos para a verificação do aumento da vazão do pasteurizador realizado pela empresa.

Palavras-Chaves: Pasteurização, Vazão e Produção.

\section{ABSTRACT}

Food production is generally practiced in all regions of Brazil, and the biggest challenge for all companies is to manufacture healthy foods with the lowest possible risk of contamination. Pasteurization is one of the procedures used to decrease microorganisms present in food. However, this study aimed to perform mechanical calculations to increase the flow of a pasteurizer in the juice and pulp company Britvic Ebba located in the city of Astolfo Dutra - MG, increasing its flow from 7860L / h to 9000L / h. Information on time, pressure and speed resulting from the process were collected, all data were verified in the process in real time of production. The results found 
demonstrated that with the fluid pressure, the outlet speed will be less than the start speed, according to the height that the pasteurizer filling tubes are known.

It is concluded that the mechanical methods of analysis were effective to verify the increase in the pasteurizer flow performed by the company.

Key words: Pasteurization, Flow rate and Production.

\section{INTRODUÇÃO}

Segundo a Associação Brasileira da Indústria de Alimentos (2020) o ramo de alimentos registrou um aumento industrial de $0,8 \%$ contra uma queda industrial de $25 \%$ na média brasileira. O crescimento do consumo das famílias dentro dos domić́lios foi umas decorrências da pandemia do novo Corona vírus, que colaboraram para um melhor desempenho do varejo em acareação com o $1^{\circ}$ semestre de 2019.

A aferição da ABIA é de um custo médio extra de produção de $4,8 \%$ devido a Covid-19. Dessa forma tem sido um desafio a mais para as empresas do setor, o contexto atual das economias brasileira e mundial. Como a perda de produção industrial reflete em média $58 \%$ do custo total do ramo, um incremento de 4,8\% neste item representa uma repercussão no valor total da indústria de alimentos entre $2 \%$ e $2,5 \%$. No primeiro semestre de 2020, as exportações de alimentos industrializados totalizaram US\$17,6 bilhões, o que configura um aumento de $12,8 \%$ em relação ao valor averiguado no primeiro semestre do ano anterior, dando suporte a demanda de mais de 180 países.

Por conta da expansão e concorrências de mercado, essas apostam em diversos métodos e estudos capazes de melhorar e ampliar seu índice de produtividade e vêm se empenhando para desenvolver técnicas de produção para o crescimento de sua lucratividade e garantir que seus produtos continuem com a mesma qualidade.

O estudo propõe o crescimento da capacidade produtiva aumentando a vazão de um pasteurizador da Britvic Ebba, empresa que hoje comercializa bebidas não alcoólicas para todo o Brasil, expandindo assim sua produção, garantindo um avanço no índice de produtividade. Contudo ter maior quantidade de produto acabado em estoque, cresce a importância das entregas em tempo record e como principal destaque o ganho de lucro com o material e como consequência a não obtenção de maiores custos. Do mesmo modo, mostrar por meios de estudos por cálculos mecânicos como o fluido reage dentro dos tubos do pasteurizador com a nova vazão, respeitando o fluxo padrão estabelecido pela empresa. 
O objetivo do presente artigo é calcular o Coeficiente de Reynolds, para descobrir se o regime de escoamento dos holdings são laminares ou turbulentos, bem como se os tubos laminares e o suco tendem a passar com menos atrito, já que tubos turbulentos tendem a passar o suco com mais atritos. Dessa forma usou-se cálculos mecânicos apresentando as perdas de carga que o fluido sofrerá em cada curva existente no pasteurizador, com o aumento da vazão de $7860 \mathrm{~L} / \mathrm{h}$ para uma vazão de $9000 \mathrm{~L} / \mathrm{h}$. Ao final, com a utilização do cálculo de Bernoulli permitiu-se enxergar a variação de velocidade que o suco terá da sua entrada no processo até a sua saída. O aumento dessa vazão, e a saída de PA (Produto Acabado), será drasticamente maior, alcançando um maior volume de suco por hora, gerando lucros significativos para a empresa com o menor custo, sendo que suas demandas serão entregues no tempo necessitado pelo cliente.

\section{REFERÊNCIAL TEÓRICO}

\subsection{Processo Produtivo}

De acordo com Passarini (2018), o processo produtivo com sua aplicação, objetiva analisar e avaliar a estabilidade e a capacidade de um sistema produzir resultados dentro dos níveis possíveis, ou mesmo extrapolar os atuais resultados conquistados. Esses processos obtidos passam por diferentes etapas como a implantação da padronização do gerenciamento produtivo, análises gráficas, fluxograma de processos que o propósito seja a identificação dos gargalos e a resolução dos mesmos.

Segundo Lira (2017), no entendimento sobre o processo produtivo é permitido que se tenha o poder de decisão e visão ampla empresarial, de vários cenários que podem atrapalhar os proveitos conquistados, como a redução de custos e prazos de entregas.

\subsubsection{Capacidade Produtiva}

Para Penof et al (2017), a capacidade produtiva é uma das preocupações dos gestores de produção que buscam resolver e cumprir as expectativas da demanda atual e futura.

“A capacidade de um processo é o máximo de produção que se pode alcançar com o valor agregado em estabelecido período de tempo em que o sistema efetua nas condições normais, além do planejamento e controle da capacidade efetiva da execução produtiva e assim responder com eficácia a demanda de mercado". (PENOF) et al, 2017, p. 41.

2.1.2 Linha de Produção 
Para Gouvea (2019), a linha de produção é uma busca constante de excelência para diferentes ramos de produtos. Esses produtos passam por diferentes processos e com diferentes máquinas e operadores, mas além desses fatores o que faz a produção ser qualitativa e quantitativa é a forma de gestão por parte da liderança e adesão de novidades que aparecem no mercado.

\section{METODOLOGIA}

\subsection{Empresa Objeto de Estudo}

O presente estudo foi realizado na empresa Britvic Ebba, formada em 2009, tornou-se uma das maiores companhias do mundo no setor de bebidas não alcóolicas, com o propósito de unir as maiores marcas de suco do país. Hoje sua linha contempla unidades industriais em Minas Gerais e Ceará, possuindo uma capacidade produtiva de aproximadamente 200 milhões de litros de suco por ano, uma organização que se destaca como uma empresa resistente e competitiva e que possui habilidade em todo o processo produtivo, desde o acompanhamento da colheita dos frutos, até o envase da fruta já processada, possuindo controles que garantem produtos desejáveis de alta qualidade.

\subsection{Objeto de Estudo}

O objeto estudado para o desenvolvimento do projeto foi o pasteurizador, equipamento que é utilizado para destruir microrganismos existentes em alimentos.

Este processo baseia-se no aquecimento do alimento a uma determinada temperatura e por um determinado tempo. Depois de aquecido, o alimento é resfriado a uma temperatura inferior a de início, eliminando os micro-organismos ali presentes, além de conservar os produtos por mais tempo, facilitando um método de produção de alimentos mais seguros. A figura 1 apresenta o fluxograma do processo de pasteurização do suco:

\subsubsection{Fluxograma do Processo do Pasteurizador}




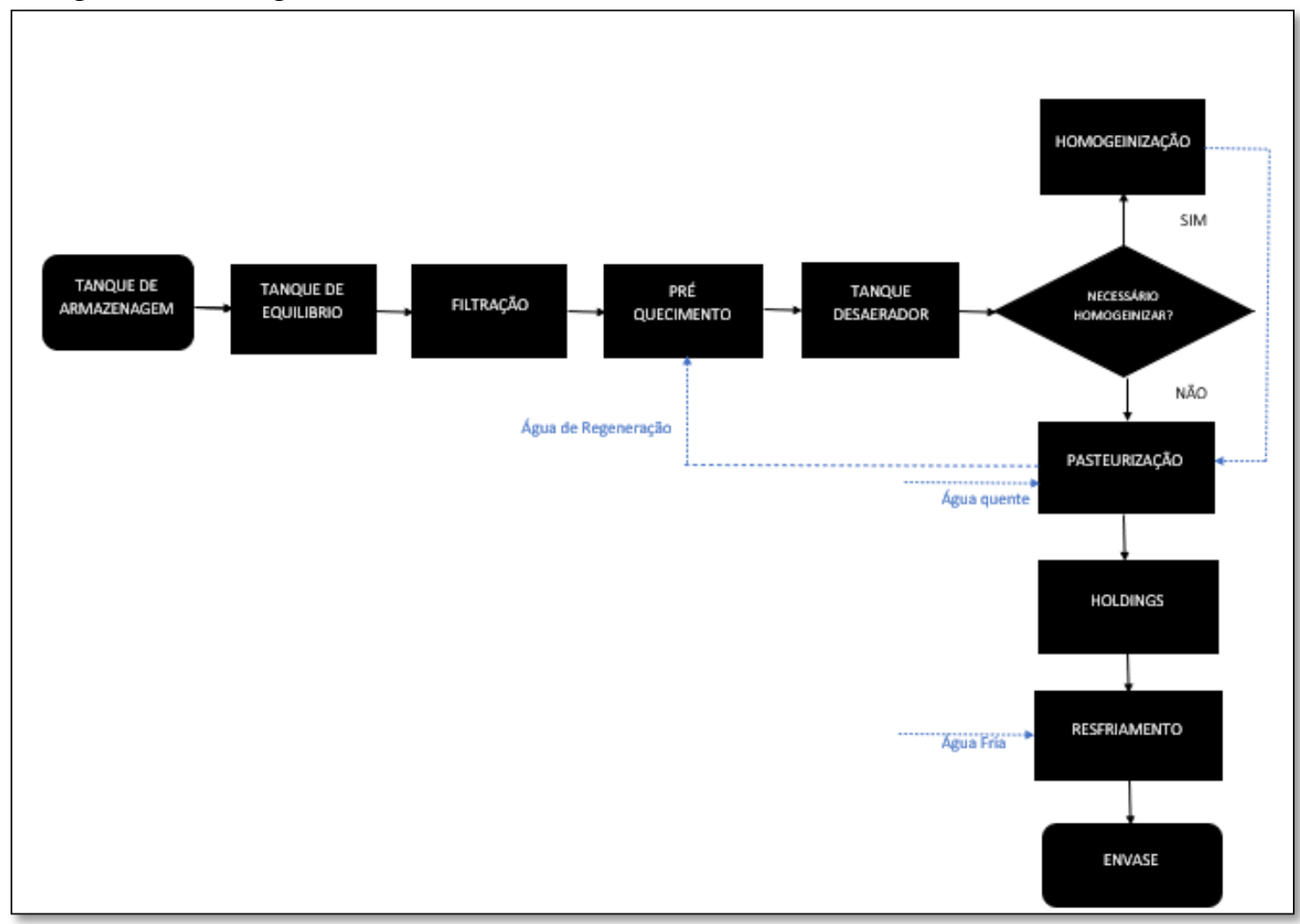

Fonte: Próprios Autores (2020)

\subsubsection{Pasteurizador}

Para dar início ao processo, as polpas chegam armazenadas em tambores e são jogadas no tanque de formulação e misturadas com outros insumos. O formulado é destinado para o Tanque de Armazenagem (11 mil L) a uma temperatura ambiente onde é batido constantemente. Finalizando o processo de formulação, o suco fica armazenado e, em poucas quantidades, destina-se para o tanque de equilíbrio, tanque este que recebe o suco do tanque de Armazenagem e os retornos de linha, por isso dá-se nome de " tanque de equilíbrio", uma vez que mantém o equilíbrio da linha ligada ao pasteurizador e mantém o equipamento sempre cheio na vazão de trabalho.

Logo após, o suco é submetido ao processo de filtragem, na qual dois filtros (de até $1 \mathrm{~mm}$ ), garantem que nenhum corpo estranho passe a diante para ser pasteurizado. Em seguida, inicia-se o processo de pré-aquecimento, em que o produto se encontra indiretamente com a água da secção regenerativa, seguindo pelo desaerador que tem por finalidade principal retirar a maior quantidade de ar do produto, que provocam alterações de cor, aroma e sabor. 
A próxima etapa é a homogeneização, o produto é sujeito a altas pressões, fazendo com que quebre as partículas em tamanho ainda menores e fique homogêneo, ajudando no processo indesejável de separação de fases. Do homogeneizador o produto segue para área de aquecimento onde o produto atinge uma temperatura de $98^{\circ}$, entrando para uma fase de "repouso", o Holding, ficando nesta área por 30 segundos é realizado o processo de pasteurização.

Em seguida, o produto segue seu curso para o pré-resfriamento que recebe água da regeneração (água que trabalha trocando temperatura, com os módulos de préresfriamento, ganhando temperatura vinda da pasteurização, e em seguida vai para o préaquecimento e perde temperatura, pois o produto está em uma temperatura ambiente, fazendo um ciclo permanente). Após esta etapa o produto entra nos módulos de água gelada, atingindo uma temperatura de envase próximo dos $25^{\circ}$, e por fim é direcionado para o setor de envase.

\subsection{Cálculo de Reynolds}

Reynolds, por meio do seu trabalho, descobriu um número adimensional que prevê o fluxo de um fluido com base nas propriedades estáticas e dinâmicas, como densidade, velocidade, viscosidade dinâmica e características dos fluidos. Ele acompanhou estudos práticos para explorar a relação entre a velocidade e o comportamento dos fluidos, e descobriu que a razão entre forças inerciais e forças viscosas também pode ser vista como uma capacidade de forças de cisalhamento turbulentas e forças de cisalhamento laminar.

Com base em sua experiência, determinou-se um número capaz de classificar os escoamentos como laminar, de transição, ou turbulento entre as formas, número chamado "número de Reynolds $(\mathrm{Re})$ ".

Com a finalidade de relacionar cada tipo de escoamento ao número de Reynolds, verificou-se que, no caso de tubos, seriam observados os seguintes valores:

$\mathrm{RE}<2.000$ Escoamento Laminar

$2.000<\mathrm{RE}<2.400$ Escoamento de Transição

RE > 2.400 Escoamento Turbulento

A princípio foi transformada a nova vazão desejada a ser pasteurizada de 9000litros/hora para metros cúbicos/segundo. Logo após, foi calculada a velocidade que 
os fluidos passariam nos tubos do pasteurizador. Para realizar esse cálculo, foi dividida a nova vazão sobre a área dos tubos, onde o suco percorre dentro do pasteurizador.

Como padrão da empresa o regime de escoamento no pasteurizador precisa ser laminar, e para garantir que mesmo com o aumento da vazão seu regime será o mesmo, obedecendo adequadamente às especificações de qualidade, foi necessário desenvolver o cálculo de Reynolds, seguindo a seguinte fórmula:

$$
\mathrm{Re}=\frac{\rho \mathrm{DD}}{\mu}
$$

Re: número de Reynolds;

$\rho$ : massa específica;

v: velocidade do fluido;

D: diâmetro;

$\mu$ : coeficiente de viscosidade.

Para a execução desse cálculo, foram coletadas as medidas dos tubos do pasteurizador, para a sua capacidade produtiva o valor é de 9000 litros/hora que foram transformados para $0,0025 \mathrm{~m}^{3} / \mathrm{s}$. Seguindo, foram levantados dados mediante a polpa mais densa, hoje representado pela goiaba com sua viscosidade $0,2 \mathrm{~kg} / \mathrm{m}$.s e sua massa específica equivalentes à $1006 \mathrm{~kg} / \mathrm{m}^{3}$. O cálculo da fórmula foi realizado de acordo com os dados colhidos na empresa e transformados na unidade de medida correta.

\subsection{Regime Turbulento}

O escoamento turbulento apresenta partículas que estão em deslocamento caótico macroscópico, isto é, a aceleração aponta elementos transversais ao movimento geral do grupo ao fluido. $\mathrm{O}$ escoamento turbulento mostra também algumas propriedades que são importantes como a irregularidade, difusividade, altos números de Reynolds, flutuações tridimensionais, dissipação de energia, conforme mostra a figura 2:

Figura 2- Escoamento turbulento

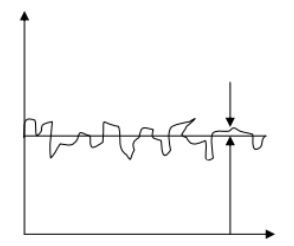

Fonte: SILVEIRA, Neto (2012)

\subsection{Regime Laminar}


Esse escoamento laminar é determinado como o fluido que se movimenta em nível, ou lâminas, uma camada decorrendo sobre adjacente, assim tem somente a troca de partes do movimento molecular. Caso houver oscilações na instabilidade e turbulência, os mesmos são amortecidos pela força viscosa de cisalhamento que impossibilitam a deslocação relativa entre as camadas adjacentes do fluido. A metodologia de um escoamento é laminar ou turbulento e sua colocação relativa em escala de turbulência é apresentada pelo número de Reynolds que indica a relação entre força de inércia e força viscosa, como é mostrado na figura 3:

Figura 3- Escoamento Laminar

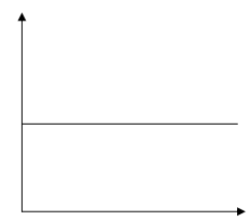

Fonte: SILVEIRA, Neto (2012)

\subsection{Equação Hazen Willians}

O transporte de um fluído no interior das tubulações sofrem interferências nas paredes no seu interior, devido a sua rugosidade, gerando perdas de carga no processo. A equação de Hazen Willians foi utilizada para permitir simplificação do cálculo da perda de carga no escoamento do suco de goiaba nas tubulações de seção circular da empresa em estudo.

A equação de Hazen-Willlians foi inicialmente calculada com o coeficiente de rugosidade "C" de 150 para tubulação de Aço Inox 316, material do pasteurizador, em seguida foi inserido na fórmula a vazão de $0,0025 \mathrm{~m}^{3} / \mathrm{s}$, e por fim utilizou-se os diâmetros dos tubos com o valor de 0,015875 vezes 12 "número de furos internos que o suco percorre dentro dos tubos" tendo um diâmetro de $0,1905 \mathrm{~m}$. Com todos esses dados, temse a fórmula abaixo:

$$
J=\frac{10,622 \cdot Q^{1,85}}{C^{1,85} \cdot D^{4,87}}
$$

$\mathbf{J}=$ perda de carga unitária $(\mathrm{m} / \mathrm{m})$;

$\mathrm{Q}=$ vazão de escoamento $\left(\mathrm{m}^{3} / \mathrm{s}\right)$;

$\mathrm{C}=$ coeficiente de rugosidade (adimensional);

$\mathrm{D}=$ diâmetro (m). 
Através do desenvolvimento da equação foi verificado a vazão, a quantidade de curvas simultaneamente e o comprimento dos tubos, fornecendo parâmetros para a seleção da bomba correta para o processo.

\subsection{Equação universal para a perda de carga}

Para instalações hidráulicas no regime de conduto forçado, é significativo que o suco chegue no ponto de consumo com a vazão e a pressão necessárias. Para analisar se o sistema irá atender a esses dois fatores, é de suma importância calcular a perda de carga nas tubulações, que variam de acordo com a vazão de desempenho do processo.

A mesma tem por princípio calcular a perda de carga em tubos transportando fluidos, sendo estes líquidos. Conforme o suco escoa ao longo dos tubos a velocidade diminui devido as curvas que os tubos apresentam, e para calcular em metros quanto de carga o suco perde em cada curva foi utilizado a fórmula a seguir:

$$
H=\frac{f L V^{2}}{D 2 g}
$$

$\mathrm{H}=$ Perda de carga total, em m;

$\mathrm{f}=$ Coeficiente de atrito da tubulação, adimensional;

$\mathrm{L}=$ Comprimento da tubulação, em m;

$\mathrm{V}=$ Velocidade de escoamento, em $\mathrm{m} / \mathrm{s}$

D = Diâmetro da tubulação, em m;

$\mathrm{g}=$ Aceleração da gravidade, $\mathrm{em} \mathrm{m} / \mathrm{s}^{2}$.

Para o início do cálculo da perda de carga na tubulação do pasteurizador utilizouse o coeficiente de atrito "f" 0,024 (adimensional), com o diâmetro de 0,1905m e a gravidade padrão de $9,81 \mathrm{~m} / \mathrm{s}^{2}$. Desta forma realizou-se um somatório do comprimento de escoamento "L" em metros de todos os tubos e curvas do pasteurizador no qual, o pasteurizador possui, 49 tubos de $6 \mathrm{~m}$ cada, 48 curvas de 66m, 4 curvas com $75 \mathrm{~m}$ e por fim 6 curvas de holdings com 33cm totalizando 330,66m de comprimento. Dados "J" da Hazen Willians cálculo feito acima foi multiplicado seu resultado pelo comprimento da tubulação obtendo o total da perda de carga "H", e para finalização do cálculo evidenciouse a velocidade do escoamento "V" $\mathrm{em} \mathrm{m} / \mathrm{s}$.

\subsection{Método Bernoulli}


A equação de Bernoulli é necessária para relacionar a pressão do fluido, velocidade e elevação do mesmo em um regime permanente do escoamento. A polpa de goiaba sendo a mais densa, dessa forma a equação traz o valor da velocidade com que o fluido passa no pasteurizador e seu comportamento qualitativo que causa esse efeito de redução da pressão e o aumento da velocidade em algumas partes da tubulação do pasteurizador.

Portanto analisou-se a pressão que o pasteurizador trabalha com uma força de 400.000Pa, depois foi verificado a diferença de densidade da goiaba quando ela entra no pasteurizador e quando ela sai, com a entrada de $1002 \mathrm{~kg} / \mathrm{m}^{3}$ e saída de $1006 \mathrm{~kg} / \mathrm{m}^{3}$, utilizou-se a gravidade de $9,81 \mathrm{~m} / \mathrm{s}^{3}$, foi medido a altura do chão até o tubo final em que o suco sai do pasteurizador com destino ao envase "H1", e a altura do chão até a bomba de pressão "H2" em metros. Com a velocidade apresentada com o cálculo acima, da perda de carga, utilizou-se V1 como velocidade de início, e por meio da fórmula a seguir estudou-se a diferenciação de velocidade que o fluido apresentará no processo "V2" em m/s.

$$
P_{1}+\frac{1}{2} \rho v_{1}^{2}+\rho g h_{1}=P_{2}+\frac{1}{2} \rho v_{2}^{2}+\rho g h_{2}
$$

P1: Pressão de trabalho 1;

P2: Pressão de trabalho 2;

$\mathrm{g}$ : Aceleração da gravidade, em m/s²;

$\rho 1$ : Massa específica de entrada;

ค2: Massa específica de saída;

h1: Altura do pasteurizador;

h2: Altura da bomba;

V1: Velocidade de entrada;

V2: Velocidade de saída.

\section{RESULTADOS E DISCUSSÕES}

O objetivo do estudo é aumentar a vazão em litros por hora de um pasteurizador em uma empresa de suco, obedecendo o regime de escoamento padrão da empresa, e mostrar como o fluido reagirá dentro dos tubos com a nova vazão. Portanto toda a metodologia está baseada nos cálculos de Hazen Willians, Equação de Perda de Carga e 
Bernoulli para apontar se a vazão pode ser aumentada e se os tubos irão suportar a quantidade de suco.

Para a produção do aumento da vazão de 90001/h o valor encontrado no cálculo de Reynolds é de 84,04503 evidenciando que o regime de escoamento continua sendo laminar, mantendo o padrão da empresa Britvic EBBA. Em seguida, com cálculos de Hazen Willians verificou-se o somatório de perda de carga em que o suco perde $5 \times 10^{\wedge}-5$ $\mathrm{m} / \mathrm{m}$ de carga, com os atritos da rugosidade dos tubos e suas curvas. Logo após foi utilizado a Equação Universal da Perda de Carga e analisou-se que em cada curva dos tubos o suco perde $0,0882 \mathrm{~m} / \mathrm{s}$ de velocidade. A diferenciação de velocidade do fluído com a nova vazão usando a pressão de trabalho do pasteurizador foi representado pela equação de Bernoulli, expondo que a velocidade de saída do suco para a envasadora é de $6,614 \mathrm{~m} / \mathrm{s}$.

Com a validação da metodologia demonstrada por meio dos cálculos citados acima, pretende-se aumentar a vazão de 7860 para 9000 litros por hora, para que se identifique o comportamento do pasteurizador nesse novo modelo simulado, e assim obter os resultados desejados. Com essa aplicação pretende-se um aumento no índice de produtividade, portanto gerando produto acabado em estoque garantindo assim uma entrega mais rápida das demandas da empresa.

\section{CONCLUSÃO}

O presente artigo foi baseado no estudo do aumento da capacidade produtiva de um pasteurizador de suco da empresa Britvic EBBA, onde hoje a vazão do suco é de 7860L/h e o objetivo é atingir 9000L/h. Dessa forma, no início do estudo foi feito a identificação do número de Reynolds e assim o resultado encontrado foi classificado como regime laminar. Em seguida foi calculado a perda de carga em cada curva do pasteurizador pela fórmula de Hazen Willians, para que com o resultado obtido fosse calculado o somatório da perda de carga com a equação universal identificada como perda de carga total. Sendo assim todos os resultados obtidos até o momento foi relacionado com a pressão do fluido através da fórmula de Bernoulli em que a velocidade de saída é menor que a velocidade de início, pois os tubos de saída têm uma altura maior que de entrada. 


\section{REFERÊNCIAS BIBLIOGRÁFICAS}

Associação Brasileira da Industria de Alimentos. Indústria de alimentos cresce $\mathbf{0 , 8 \%}$ em faturamento no primeiro semestre 2020. 12/08/2020. Disponível: https://abia.org.br/releases/industria-de-alimentos-cresce-08-em-faturamento-noprimeiro-semestre-2020.

FERNANDES, Andre; FERREIRA; Cristiano; MARTINS, Patricia; NEVES, Romina. Métodos e projetos hidráulicos e de saneamento, volume 1. UNIBE. Universidade de Uberaba, 2013, p.17 ao20.

FISMAN, Raymond; SULLIVAN, Tim. A Organização: Entenda os Bastidores das Empresas. Ed: Elsevier Editora Ltda, p. 86, 2017.

GOUVEA, Marcelo. Qualidade da linha de produção: sua empresa mantém excelência em todas as etapas? 2019. Disponível em: produza.ind.br/gestao/linha-deproducao/ Acesso em: 25 de abril em 2020.

LIRA, M. Valdemir. Princípios dos processos de fabricação utilizando metais e polímeros. 1. Ed: Edgard Blucher Ltda, p. 26 e 34, 2017.

OLIVEIRA, A. Marcos. Estratégia empresarial e gestão da informação gerencial. 2. Ed. Senac, 2018.

PASSARINI, R. Giuseppe. Gerenciamento de processos produtivos através de abordagem sistêmica. 1. Ed: Senai - SP, p.60 e 65, 2018.

PENOF, G. David; MELO, C. Edson; LUDOVICO, Nelson. GESTÃo DA PRODUÇÃO E LOGÍSTICA - Série Gestão Empresarial. 1. Ed. Saraiva, p.35 e 41, 2017.

SILVEIRA,NETO. Fundamentos de Turbulência. USP, 2012. http://www2.eesc.usp.br/netef/Oscar/FUNDAMENTOS_DE_TURBULENCIA.pdf. 


\section{APÊNDICE A- Carta para Autorização de Pesquisa}



CARTA PARA AUTORIZAÇÃO DE PESQUISA

Solicitamos autorização para realização da pesquisa intitulada Aumento da copacidade produtiva de uma empriva de seco, atrawís do aumunto da vajäo ma quantidadu em litros por hora de um pastuirugador. pelas (os) acadêmicas (os) de graduação Andrua Morais Aprigio sob orientação do Professor responsável,

thiage Bittincourt com objetivo de coletar dados para o trabalho de TCC, necessitando portanto, ter acesso aos dados a serem colhidos no setor de da empresa Brituric Ebba

Ao mesmo tempo, pedimos autorização para que o nome desta instituição conste no relatório final, bem como futuras publicações em eventos e periódicos científico. Ressaltamos que os dados coletados serão mantidos em absoluto sigilo. Salientamos ainda que tais dados serão utilizados somente para a realização deste estudo ou serão mantidos permanentemente em um banco de dados de pesquisa, com acesso restrito, para utilização em pesquisas futuras. Na certeza de contarmos com a colaboração e empenho desta Diretoria/Coordenação agradecemos antecipadamente a atenção, ficando à disposição para quaisquer esclarecimentos adicionais que se fizerem necessários.

Cataguases- MG,

04 de

mais de 2020.

(X) Concordamos com a solicitação

( ) Não concordamos com a solicitação

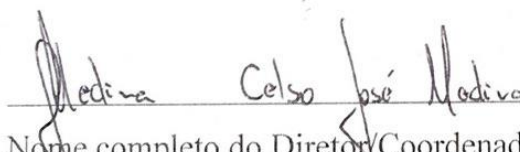

Nonge completo do Diretor/Coordenador/Chefe

Diretoria da Instituição onde será realizada a pesquisa 